\title{
The method of processing the droplet-air flow by non- equilibrium plasma
}

\author{
Alexandre Bystrov ${ }^{1, *}$, Konstantin Vostrov ${ }^{1}$, Vladimir Frolov ${ }^{1}$, and Anatoli Bistrov² \\ ${ }^{1}$ Peter the Great St. Petersburg Polytechnic University, 195251 St. Petersburg, Russia \\ ${ }^{2}$ AnaBis Solutions, 01200 Vantaa, Finland
}

\begin{abstract}
The paper considers a fundamentally new installation for the processing of a droplet-air environment in a non-equilibrium plasma. Its various designs are presented. This installation is built on the basis of pulsed energy devices and is intended for water disinfection and decomposition of dissolved organic compounds, the production of nanomaterials, research in physics and chemistry, etc. We have conducted research of the performance and energy efficiency of this method of processing. It is established that in obtaining the target product, this method of processing has an advantage over the known methods: due to high performance and energy efficiency, as well as the simplicity of this processing method. In addition, the parasitic load capacity of the nanosecond generator is reduced several times, this fact increases the voltage rise rate on the cells of the plasma plasmatrons, which improves performance and energy efficiency.
\end{abstract}

\section{Introduction}

In the modern industry, the various methods for producing a nonequilibrium plasma are existing. The main methods are carried out by means of the following types of discharges: a glow discharge, a discharge with a dielectric barrier, a corona discharge, and the same pulsed discharges [1-4]. In recent years, a method has been developed to produce non-equilibrium plasma by means of high-voltage nanosecond pulses generated by pulsed energy devices [5-7]. This method of obtaining a non-equilibrium plasma was very effective. The plasma density in the discharge increases by an order of magnitude in a few nanoseconds. The energy invested in the discharge, almost all is spent on the excitation of atoms. Unlike traditional discharges, the temperature of ions in the cathode layer does not change [8]. Inert gases - argon, helium, and also air — are most often used as the plasma-forming gas.

A very promising method is the treatment of dropletair flow with non-equilibrium plasma, which is created directly in the processed liquids and gases or on the surface of the treated objects. The interaction of active particles obtained in a liquid and gas with chemical compounds or microorganisms leads to their destruction. Today, there are many methods for treating droplet-air flow with non-equilibrium plasma [9-11]. To increase energy efficiency, it is necessary to ensure the generation of active particles directly in the processed fluid. This condition can be achieved by direct contact of a liquid drop and an electric discharge plasma. Research in this direction is associated with the use of high-voltage nanosecond pulses in a droplet-air flow. The main area of these studies is water purification [9-11]. It was established that the treatment of a droplet-air flow with non-equilibrium plasma causes disinfection and decomposition of dissolved organic compounds, and both drinking and wastewater can be purified [12]. The main disadvantage of the known methods is rather low energy efficiency. The tasks of modern traditional energy are well represented in various scientific publications [13, 14]. However, pulsed energy is designed to solve other problems, it works on fundamentally different loads. The development of conventional energy is accompanied by the creation of well-known devices, which are presented in articles [14, 15]. Devices built on the basis of pulsed energy allow you to create energy-efficient technologies. On the other hand, the further development of traditional energy is carried out using digital devices [16, 17]. The use of digital devices in pulsed energy is problematic and sometimes impossible. The development of this energy required the creation of special pulse generators, electronic keys, lines for the transfer of energy, plasmatrons, etc.

The purpose of this work is to develop an energyefficient method for treating a droplet-air flow with a nonequilibrium plasma generated by pulsed energy devices of various designs. Find out how the method of treating a drip-air stream with a nonequilibrium plasma affects energy efficiency and productivity.

\footnotetext{
* Corresponding author: stalex81@yandex.ru
} 


\subsection{On the reasons for the low energy efficiency of known methods for treating a droplet-air flow with a nonequilibrium plasma}

A pulsed barrier discharge in a droplet-air flow is by far the most effective of the existing methods for treating a droplet-air flow with non-equilibrium plasma [11].

Short-lived active particles are formed in the zone of contact between water and plasma: free radicals, ions and electrons, many of them play an important role in the processes of interaction with chemical compounds or microorganisms, which leads to their destruction and water purification. On the other hand, due to the short lifetime of active particles, most of them are not involved in the processes of interaction with chemical compounds or microorganisms. Ozone only partially transfers plasma energy into the water. $[9,11]$. With some organic compounds, ozone reacts poorly or does not react at all. It was established that the predominant factor, the destruction of chemical compounds and microorganisms in the droplet-air flow, is plasmaproduced oxidizing agents - $\mathrm{OH}^{-}$hydroxyl radicals, atomic oxygen $\mathrm{O}$, and, to a lesser extent, ozone $\mathrm{O}_{3}$.

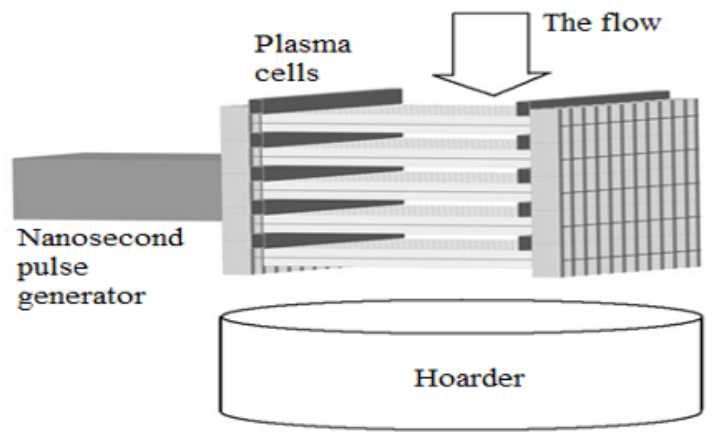

Fig. 1. Conventional setup for implementing known methods for treating a droplet-air flow with non-equilibrium plasma

The concentration of $\mathrm{OH}$ radicals increases linearly with increasing plasma energy, frequency, and amplitude of voltage pulses [12].

The geometry of the apparatus for implementing the known methods for treating the droplet-air flow by nonequilibrium plasma is shown in Fig. 1 . The sprayer, not shown in the figure, allows the dispersed solution to be dispersed above the reactor. Thus, a droplet-air flow is created, passing through a nonequilibrium plasma, where it then interacts in the discharge zone with the plasma. The discharge zone consists of several cell levels collected from the same typed plasmatrons as shown in Fig. 2.

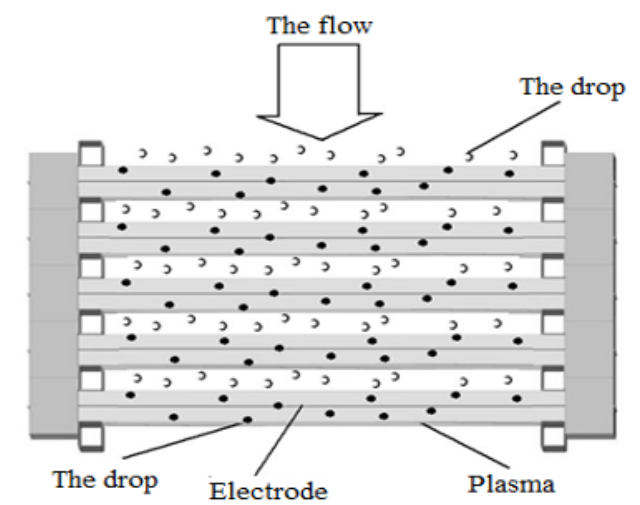

Fig. 2. The discharge zone of several levels of cells assembled from the same type of plasma torches

Hydroxyl radicals and atomic oxygen are formed directly on the surface of the water, which allows reactions with organic substances in the water to occur. The application of methods for treating a droplet-air flow with a nonequilibrium plasma does not end there. Methanol formation was detected upon treatment with a nonequilibrium plasma of a mixture of water droplets and methane [18].

Figure 2 shows the distribution of droplets at the time of the supply of a high voltage pulse to the plasma torch. It can be seen from the figure that not all drops are in the plasma exposure zone. Thus, during one passage of droplets through the plasma treatment zone, all the droplets receive a different amount of energy, and some droplets may not get into the plasma at all. This effect is especially pronounced when treating a drip-air flow with a nonequilibrium plasma generated by devices based on pulsed energy. The reason for this is the fact that the pulse duration is very short less than 50 nanoseconds, and the duty cycle is less than 0.0001. This is the main reason for low energy efficiency. Therefore, this processing method is not suitable in pulsed energy devices-based setups. In addition, to increase productivity, the number of rows of cells from plasmatrons is supposed to be increased, which entails an increase in the capacitive load of the nanosecond generator and, as a result, a further decrease in productivity and energy efficiency.

\section{Advantages of the method of processing droplet-air flow by a nonequilibrium plasma generated by pulsed energy-based devices}

The main difference between this method and the known ones is the movement of the droplet-air flow along the electrodes of the plasma torch as shown in Fig. 3a, as well as the absence of dielectric barriers in the plasma torches. The duration of the plasma column in the plasmatron is only a few tens of nanoseconds. Therefore, the contraction of a volume discharge simply does not have time to occur and the discharge gap does not cross [5]. Despite the fact that ordinary water has conductivity, 
and this fact negatively affects the known processing methods, in the proposed method, this has no effect. When exposed to normal water high-voltage nanosecond pulses, the electric strength of water increases and its conductivity decreases. The shorter the pulse, the electric strength is higher, and the conductivity is lower [19, 20]. The distribution of droplets in the experimental setup is shown in Fig. 3b.

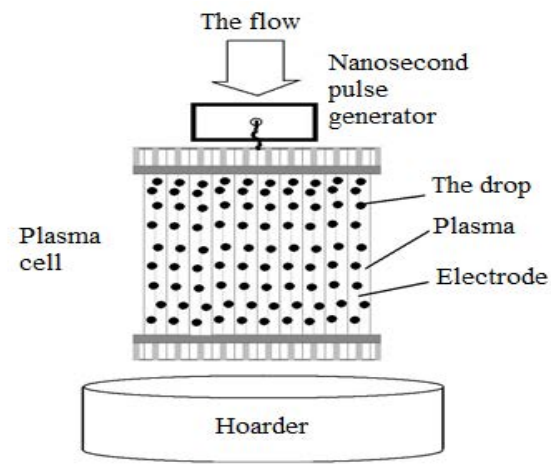

a)

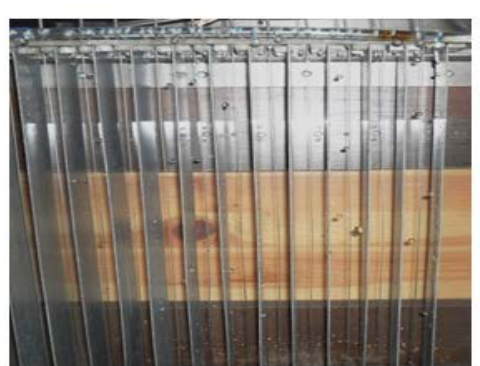

b)

Fig. 3. The setup for the implementation of a proposed method for treating a drop-air flow with a non-equilibrium plasma: a) general structure; b) droplet distribution in the experimental setup

Various designs of cells from plasmatrons are shown in Fig. 4. They differ in cross-sectional shape. The number of plasmatrons in one cell is limited by the inductance of the connecting lines of energy transfer. Cell section configurations are not limited to those shown in Fig. 4 and can vary depending on the task.

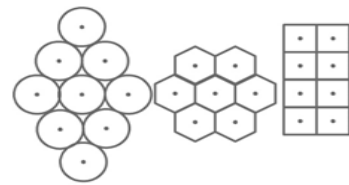

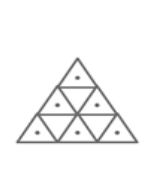

d)

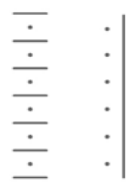

e) f)

Fig. 4. Various designs for the cross-section of plasma torch cells

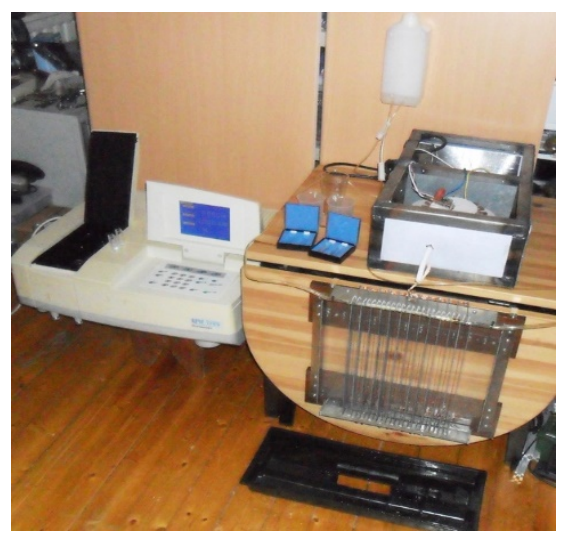

a)

\section{On The experimental part of the work}

In the experimental part of this work, the comparative characteristics of the productivity and energy efficiency of the generation of chemically active particles were estimated using the known method [21-24] shown in Fig. 2 and the proposed method is shown in Fig. 3. In the experiment, the goal was not to obtain the best characteristics of the generation of chemically active particles. For the experiments, the setup shown in Fig. 5a was used. Plasma radiation is observed, only in the dark Fig. 5b.

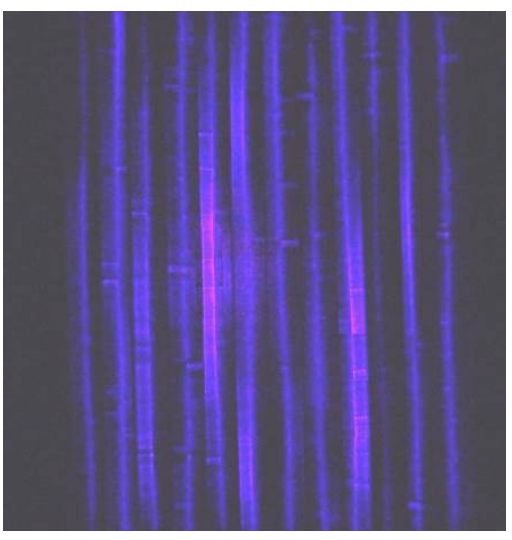

b)

Fig. 5. Photo of the experimental device: a) general view; b) plasma radiation

In order to obtain comparative characteristics of the generation of chemically active particles produced by plasma, we evaluated the effect on the decrease in the concentration of the model solution of the total number of active particles at atmospheric pressure: $\mathrm{OH}^{-}$hydroxyl radicals, atomic oxygen $\mathrm{O}, \mathrm{H}_{2} \mathrm{O}_{2}$ peroxide, and also 
ozone $\mathrm{O}_{3}$. As a model solution, a solution of potassium biphthalate in distilled water of $10 \mathrm{mg} / \mathrm{L}$ was used. The concentration of potassium bifthalate was measured by a standard Cure

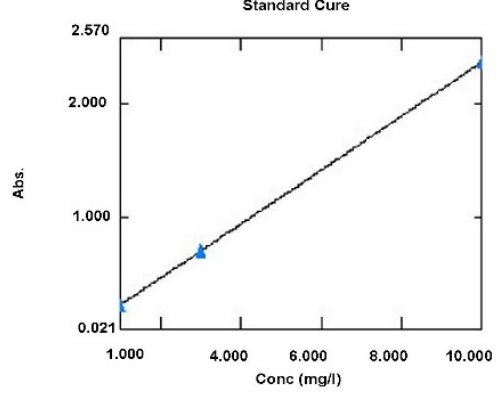

a)
Shimadzu UV-1700 dual-beam spectrophotometer. The spectrogram of the initial solution is shown in Fig. 6a.

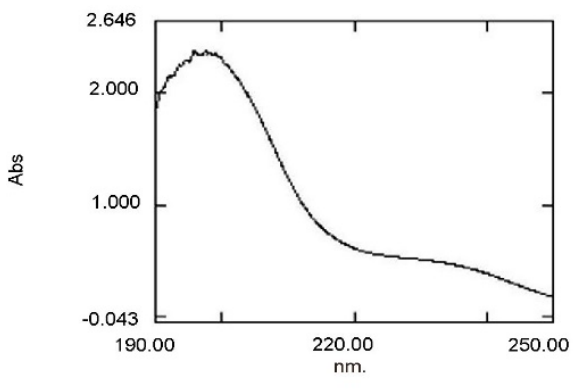

b)

Fig. 6. The spectrogram of the initial solution (a) and the calibration graph (b)

Processing the model solution in plasma lasted 20 minutes for each measurement. The experiments were carried out for two modes of the pulse repetition rate of the generator: $1000 \mathrm{~Hz}$ and $2000 \mathrm{~Hz}$. The concentration of the plasma-treated solution was determined from the measured optical density at the maximum of the spectrogram based on the calibration graph shown in Fig.6b. Samples of the treated solution were taken in 10 mm quartz cuvettes. The spectrograms of the treated solutions with plasma are shown in Fig. 7.

Since the voltage of the generator's power source is stabilized, the measurement was carried out only with changes in the generator current consumption from the power source, in the presence of a droplet-air flow and the absence of this flow in the plasma. The current was measured with a combined Agilent34401A multimeter.

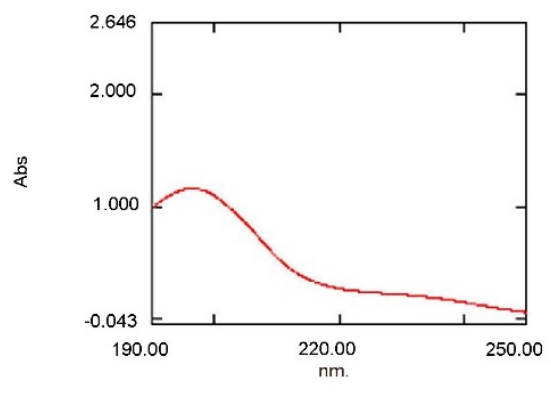

a)

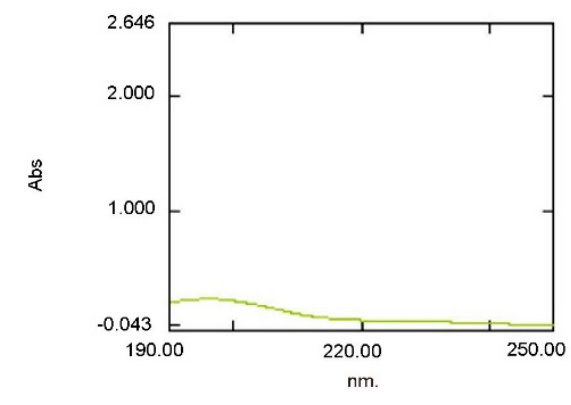

b)

Fig. 7. Spectrograms of the treated solutions with plasma: a) $20 \mathrm{~min} .1000 \mathrm{~Hz}$; b) $20 \mathrm{~min}$. $2000 \mathrm{~Hz}$;

The concentration of the solutions was measured in the "Photometry" mode. Fig. 8 shows the measurement results.

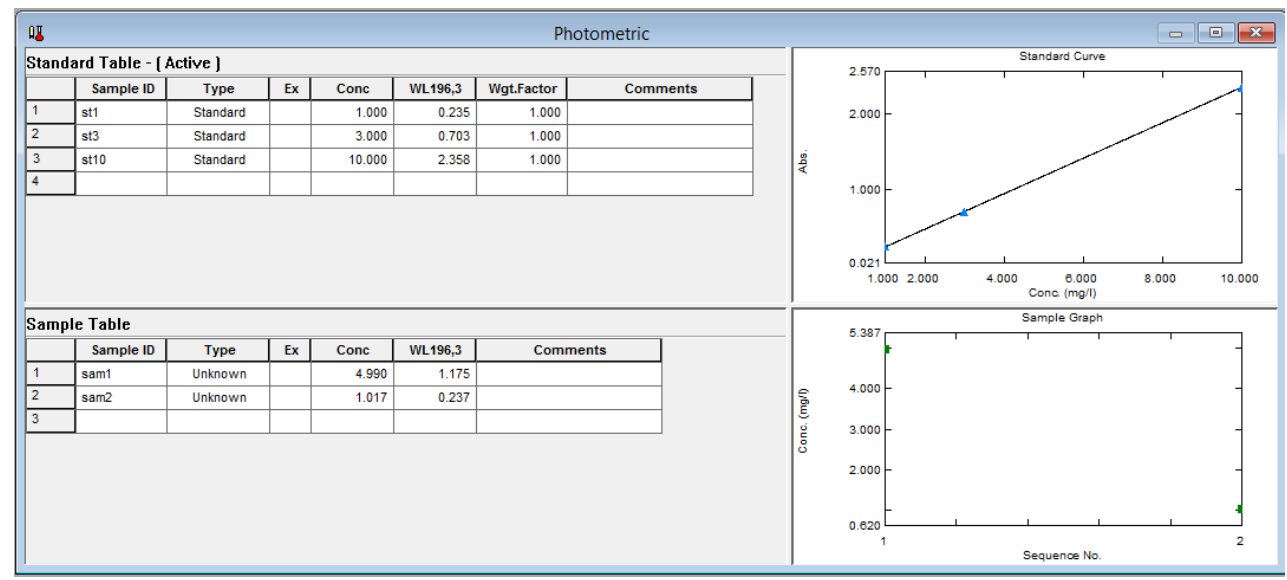

Fig. 8. The results of measuring the concentration of solutions 


\section{Measurement results and discussion}

During the work on the presented work, comparative characteristics of the generation of chemically active particles produced by plasma are obtained. All experimental data are summarized in Table 1. Based on the obtained experimental data, the values of productivity and energy efficiency are obtained. The quality relationship of the compared processing methods is very clearly visible. The table shows that at any operating condition of the generator, the proposed method for processing the droplet-air flow by nonequilibrium plasma is more productive and efficient.

Table 1. Comparative characteristics of the performance and energy efficiency of the generation of chemically active particles

\begin{tabular}{|c|c|c|c|c|}
\hline Generator & \multicolumn{2}{|c|}{$\begin{array}{c}\text { Pulse repetition rate } \\
\mathbf{1 0 0 0} \mathbf{~ H z}\end{array}$} & \multicolumn{2}{c|}{$\begin{array}{c}\text { Pulse repetition rate } \\
\mathbf{2 0 0 0} \mathbf{~ H z}\end{array}$} \\
\hline Method & Conventional & Suggested & Conventional & Suggested \\
\hline $\begin{array}{c}\text { Concentration after treatment } \\
\text { mg/l }\end{array}$ & 8 & 5 & 6 & 1 \\
\hline Electric current change, $\mathrm{mA}$ & - & 77 & - & 155 \\
\hline Relative performance in 20 minutes, mg & 20 & 50 & 40 & 90 \\
\hline Relative energy efficiency, $\mathrm{w} * \mathrm{H} / \mathrm{mg}$ & 9 & 3.7 & 9.12 & 4.1 \\
\hline
\end{tabular}

\section{Conclusion}

The proposed method for treating a droplet-air flow with a non-equilibrium plasma has high productivity and energy efficiency. In addition, the installation has a simple design and is able to isolate the plasma exposure zone from the environment. This fact expands the possibilities of this processing method. For example, it becomes possible to install in mines to reduce the concentration of methane. To date, the proposed processing method is the first and only. It is necessary to continue work in this direction to establish quantitative dependencies of the characteristics of the installation.

\section{References}

1. Yu.P. Raiser, Gas Discharge Physics, 3d ed. (2009)

2. B. M. Smirnov, Theory of Gas Discharge Plasma (2015)

3. R. B. Baksht, Y. Yankelevich, A. Pokryvailo, and V. I. Oreshkin, IEEE Trans. Plasma Sci. 34, 1725 (2006)

4. U. Kogelschatz, Plasma Chem. Plasma Process. 23, 1 (2003)

5. G. A. Mesyats, Pulsed power and electronics (2004)

6. C. Zhang, T. Shao, Z. Niu, J. Xu, H. Jiang, Y. Yu, P. Yan, and Y. Zhou, IEEE Trans. Plasma Sci. 39, 2208 (2011)
7. P.A. Bokhan, P.P. Gugin, D.E. Zakrevsky, M.A. Lavrukhin, J. of Tech. Phys. 85, 10 (2015)

8. E. A. Bogdanov, A. A. Kudryavtsev, and R. R. Arslanbekov Plasma Phys. 46, 10 (2006)

9. L. Makalskiy and O. Tsekhanovich, Vestn. Volgogr. Gos. Univ. Ser. 11. Estestv. Nauk. 7, 12 (2017)

10. T. Sugai, W. Liu, A. Tokuchi, W. Jiang, and Y. Minamitani, IEEE Trans. Plasma Sci. 41, 967 (2013)

11. J. Choi, T. Yamaguchi, K. Yamamoto, T. Namihira, T. Sakugawa, S. Katsuki, and H. Akiyama, IEEE Trans. Plasma Sci. 34, 1744 (2006)

12. A. Sugiarto, S. Ito, T. Ohshima, M. Sato, J. Skalny, J. of Electrostatics, 58, 1 (2003)

13. I. N. Voytyuk and O. M. Bolshunova, in 2019 IEEE Conf. Russ. Young Res. Electr. Electron. Eng., 736739 (2019)

14. S. P. Kuksenko, IOP Conf. Ser. Mater. Sci. Eng. 560, 012110 (2019)

15. S. V. Solovev, S. B. Kryltcov, and I. N. Voytyuk, in 2018 IEEE Conf. Russ. Young Res. Electr. Electron. Eng, 803-806 (2018)

16. V. Y. Frolov, A. V. Bystrov, and A. A. Neelov, in 2017 IEEE Conf. Russ. Young Res. Electr. Electron. Eng, 838-840 (2017)

17. V. Y. Frolov, A. A. Neelov, R. I. Zhiligotov, and A. V. Bystrov, in 2018 IEEE Conf. Russ. Young Res. Electr. Electron. Eng, 626-628 (2018) 
18. C.-J. Liu, B. Xue, B. Eliasson, F. He, Y. Li, and G.H. Xu, Plasma Chem. Plasma Process. 21, 301 (2001)

19. In Pulsed Power Springer US, Boston, MA, 91-106 (2005)

20. P. Felsenthal, J. Appl. Phys. 37, 3713 (1966)

21. M. Klavarioti, D. Mantzavinos, and D. Kassinos, Environ. Int. 35, 402 (2009)

22. S.V. Korotkov, M.Y. Andrianova, A.K. Kozlov, D.A. Korotkov, and G. L. Spichkin, Instrum Exp Tech. 62, 4 (2019)

23. A. V. Surov, S. D. Popov, E. O. Serba, V. A. Spodobin, G. V. Nakonechniy, A. V. Pavlov, A. V. Nikonov, D. I. Subbotin, V. E. Popov, and N. V. Obraztsov, J. Phys. Conf. Ser. 927, (2017)

24. Polozov, N. Razumov, T. Makhmutov, A. Silin, A. Kim, and A. Popovich, Mater. Lett. (2019) 